\title{
Revisión
}

\section{Displasia quística de rete testis: revisión de la literatura}

\author{
Raquel González López, Gonzalo Bueno Serrano, Ricardo García Navas, Víctor Díez Nicolás, \\ José Julián Vázquez Escuderos, Javier Mayor de Castro
}

Servicio de Urología. Hospital Universitario Ramón y Cajal. Madrid, España

\section{Resumen}

Objetivo: Revisión de la Displasia Quística de Rete Testis en el momento actual.

Material y Métodos: Búsqueda bibliográfica en la base de datos Medline/PubMed del término "Cystic dysplasia of the testis", con análisis de las revisiones bibliográficas encontradas.

Resultados: La displasia quística de rete testis (DQRT) es una rara enfermedad benigna, asociada a malformaciones del tracto urinario superior. Su manifestación clínica más frecuente es el aumento de tamaño testicular, demostrándose la presencia de los quistes ecográficamente. No existe consenso en el tratamiento, optándose en la mayoría de los casos por la confirmación anatomopatológica con conservación del parénquima testicular.

Conclusión: La DQRT es una enfermedad que debe ser tenida en cuenta en el diagnóstico diferencial de la masa escrotal infantil.

Palabras clave: Quistes. Testículo. Alteraciones urogenitales.

\section{Cystic displasia of the testis. Review of literature}

\section{Abstract}

Objetive: Review this pathology nowadays.

Methods: We search in Medline/PubMed database for reviews about cystic dysplasia of the testis. We review and discuss the relevant literature about it.

Results: Cystic dysplasia of the testis (CDT) is a rare benign disease, associated with upper urinary tract malformations. Its most frequent clinical manifestation is the increase of testicular size; the presence of cysts is demonstrated by ultrasound. No consensus exists in its treatment, it oftenly requires histological confirmation, performing testicle-sparing surgery.

Conclusion: CDT needs to be taken into account in the differential diagnosis of childhood testicular tumors.

Keywords: Cysts. Testis. Urogenital abnormalities.

La displasia quística de rete testis es una rara entidad benigna, frecuentemente asociada a malformaciones del tracto urinario superior, que se debe tener en cuenta en el diagnóstico diferencial de las tumoraciones escrotales de la infancia. Esta infrecuente patología supone un punto de desencuentro patogénico y terapéutico en el campo de la Urología moderna.

\section{OBJETIVOS}

Revisión de la patología en el momento actual, centrándonos en su etiopatogenia y tratamiento.

\section{MATERIAL Y MÉTODOS}

Se realiza búsqueda bibliográfica en Medline/PubMed utilizando como criterios de búsqueda:
- Dirección de búsqueda: http://www.ncbi.nlm. nih.gov/pubmed/

- Fecha de revisión: Abril de 2008.

- Periodo de revisión: desde el inicio hasta la actualidad.

- Palabras claves: cystic dysplasia of the testis.

- Idiomas: inglés y español.

Se encuentran un total de 43 artículos, de los cuáles 11 son revisiones bibliográficas. Se realiza análisis de las revisiones encontradas, desechándose aquéllas que no tratan fundamentalmente el tema y ampliándose la búsqueda a algunos artículos citados en las mismas.

Se analizan los datos encontrados referidos a etiología, clínica, diagnóstico y tratamiento de esta patología. 


\section{RESULTADOS}

Descrita por primera vez en 1973 por Leissring y Oppenheimer en un niño de 4 años con masa escrotal y agenesia renal ipsilateral ${ }^{1}$, la Displasia Quística de Rete Testis (DQRT) es una infrecuente patología benigna consistente en la aparición de quistes a nivel del mediastino testicular, de pequeño tamaño. La relevancia de esta patología se debe a dos sucesos fundamentales. El primero, su frecuente asociación con malformaciones del aparato urogenital. El segundo, la importancia de realizar un buen diagnóstico diferencial con otras causas de tumoración escrotal, como tumores testiculares, hidrocele, varicocele y otros ${ }^{2}$.

En el desarrollo embriológico del aparato urogenital, se produce la formación del conducto espermático a partir del conducto mesonéfrico de Wolf. Este conducto espermático se fusiona con el testículo y la rete testis, provenientes del seno urogenital, en la $9^{a}$ semana de vida intrauterina. La ausencia o parcialidad de esta fusión han sido postuladas por diversos autores como la causa de aparición de la DQRT ${ }^{1-10}$. Asimismo, la ausencia de inducción del blastema metanéfrico por parte del esbozo ureteral produce la agenesia o malformación del futuro riñón. Este esbozo ureteral también se desarrolla a partir del conducto mesonéfrico de Wolf, motivo por el cual se explicaría la asociación de la DQRT con las malformaciones de la vía urogenital superior ${ }^{1-10}$. Éstas se encuentran asociadas en más del 80\% de los casos, siendo la más frecuente la agenesia renal $(41 \%)^{6}$.

Además del mal desarrollo, se cree que existen causas funcionales que podrían contribuir al desarrollo de la DQRT. Así, Nistal et al. ${ }^{3}$ postulan que la ausencia o defecto de absorción por los ductos deferentes del líquido secretado en la rete testis puede producir la transformación quística de ésta, si bien no se ha podido demostrar la producción de este líquido previamente a la pubertad.

De esta manera, en los casos sin anormalidades renales, existiría un mecanismo patogénico de tipo obstructivo o mecánico y otro no obstructivo o funcional. El primer mecanismo se debería al fallo en la anastomosis de la rete testis con el conducto mesonéfrico, presentando alteraciones en el conducto deferente y el epidídimo. El segundo, en cambio, se debería a la citada ausencia de absorción de líquido en los ductos deferentes ${ }^{1,3,5}$.

Se ha demostrado que la DQRT adquirida del adulto puede estar producida por ambos mecanismos patogénicos ${ }^{10}$
Anatomopatológicamente, la DQRT se caracteriza por la aparición de formaciones tubulo-quísticas tapizadas por epitelio cuboideo y plano, similar al que se encontraría en pacientes sanos, sin encontrar atipias celulares. Los túbulos seminíferos suelen ser atróficos o incompletos, encontrándose zonas de túbulos normales. En ellos se ha demostrado espermatogénesis activa y presencia de espermatozoides intraluminales $^{3,5,7}$

La manifestación clínica más frecuente de la DQRT es el aumento de tamaño testicular (más del $50 \%$, pudiendo presentarse también como criptorquidia (35\%) y, en raras ocasiones, como dolor testicular (menos del 10\%). El testículo izquierdo es el afecto en el 53\% de las DQRT unilaterales, mostrando también predisposición izquierda las malformaciones renales (56\% de las agenesias y 55\% de las malformaciones quísticas renales). La agenesia renal es la malformación más frecuentemente asociada (41\%), encontrándose también otras anomalías como la displasia, poliquistosis, ureterohidronefrosis, reflujo vesicoureteral, megauréter y duplicación ureteral ${ }^{6-8}$. También se han descrito un caso de hipospadias penoescrotal asociado ${ }^{11}$ y un caso de múltiples malformaciones genitourinarias ipsilaterales (DQRT, quiste de vesícula seminal, agenesia renal, mal desarrollo de hemitrígono vesical y dilatación epididimaria) ${ }^{12}$.

En la exploración del paciente es frecuente encontrar un testículo aumentado de tamaño, doloroso o no, en el que los quistes pueden llegar a ser palpables. La ecografía trans-escrotal demostrará la presencia de quistes en mediastino testicular, de un tamaño entre 3 y $20 \mathrm{~mm}$, que en raras ocasiones pueden ser bilaterales, como en el caso que se presenta $^{4,7}$. Los marcadores tumorales, como la alfafetoproteína y la fracción beta de la gonadotropina coriónica humana se encuentran habitualmente dentro de límites normales ${ }^{4,6-9}$. La presencia de quistes en el testículo obliga a la realización de ecografía renal para descartar las malformaciones a este nivel que orienten el diagnóstico hacia una DQRT $^{3-10}$ (Figs. 1 y 2).

Algunos autores opinan que la simple asociación de estos hallazgos ecográficos, junto con la normalidad de los marcadores y la demostración de anormalidades mesonéfricas ipsilaterales, como malformaciones quísticas renales o de las vesículas seminales, son datos de certeza suficiente para poder realizar el diagnóstico de DQRT sin necesidad de realizar biopsia a cielo abierto ${ }^{9}$. Otros autores, sin 


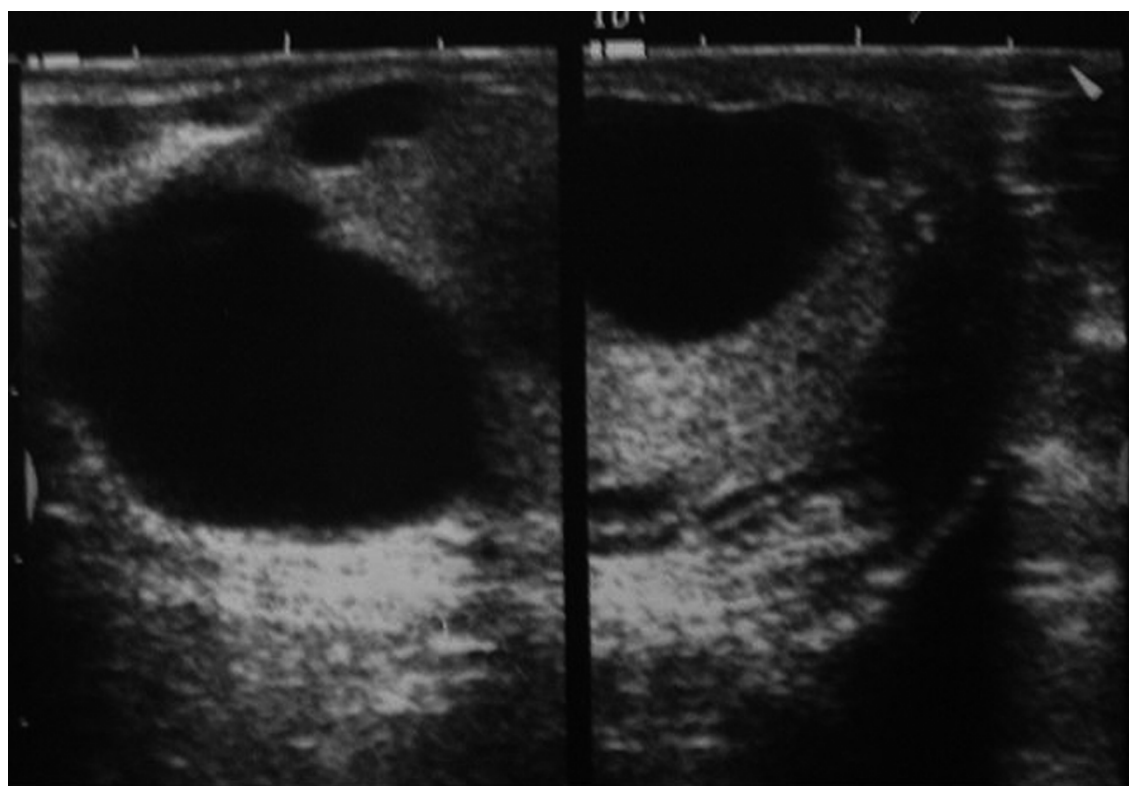

FIGURA 1. Ecografia testicular: múltiples quistes de gran tamaño, intraparenquimatosos, a nivel de mediastino testicular.

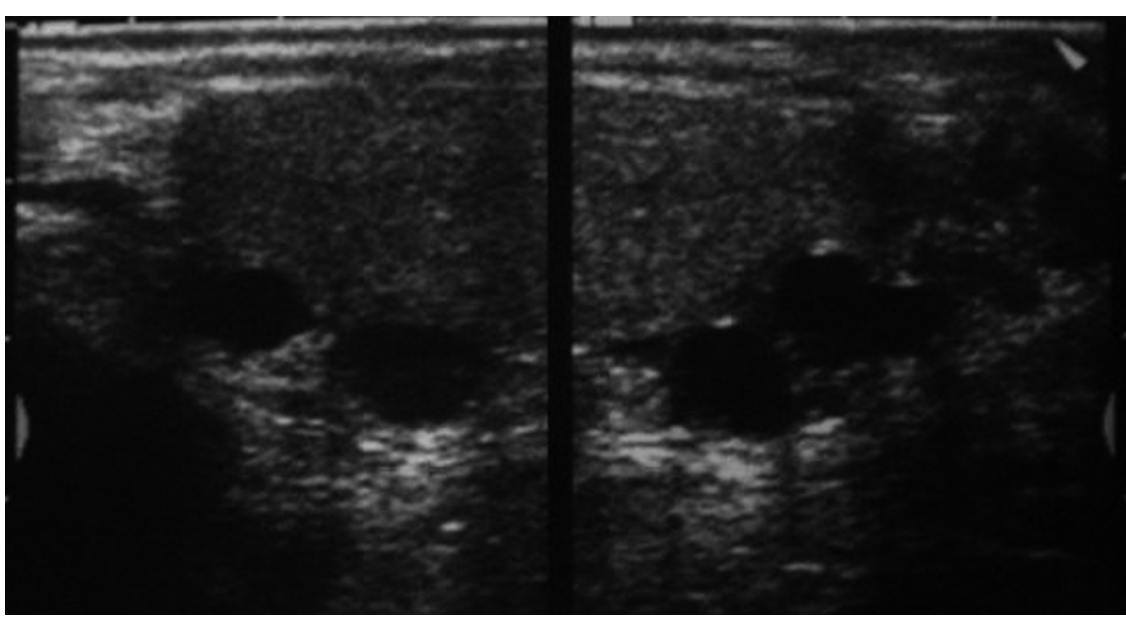

FIGURA 2. Ecografia testicular: pequeños quistes situados a nivel de rete testis.

embargo, ante la duda diagnóstica que se puede crear con determinados tumores testiculares malignos, como el seminoma, abogan por la realización de biopsia a cielo abierto o, con mayor frecuencia, orquiectomía radical del testículo afecto para análisis anatomopatológico posterior ${ }^{9,10}$.

Éste es uno de los aspectos sobre el que no existe, en el momento actual, una opinión unánime. Por una parte, algunos autores defienden el seguimiento de estos pacientes mediante ecografía periódica ( 3 meses, 6 meses y posteriormente, de forma anual), realizando orquiectomía sólo en el caso de que la lesión aumente progresivamente de tamaño o aparezca dolor ${ }^{3,7}$, mientras que otros autores abogan por la orquiectomía radical o la extirpación de la lesión ${ }^{4-6,9,10,13}$. Tal actitud se basa en la frecuencia con que esta lesión aumenta progresivamente de tamaño con el tiempo, pudiendo producir dolor en el paciente, por lo que se prefiere la extirpación en edades tempranas. Los últimos artículos al respecto abogan por la realización de cirugía conservadora de testículo, ya que la escasa edad de los pacientes y la limitación espacial de la lesión la aconsejan ${ }^{2,14}$.

No se ha demostrado, sin embargo, que la DQRT pueda sufrir degeneración maligna, así como tampoco se ha asociado a esterilidad, a pesar de la compresión del parénquima testicular que se ha demostrado en algunos $\operatorname{casos}^{3-10}$.

\section{CONCLUSIÓN}

La DQRT es una rara patología benigna testicular, más frecuente en la edad infantil ${ }^{15}$. Su manifestación clínica más frecuente es el aumento de tamaño escrotal, estando asociada a malformaciones de la vía urinaria alta en cerca del 50\% de los casos. El diagnóstico de sospecha se realiza mediante ecografía. No existe consenso en el momento actual acerca del tratamiento, abogándose en los últimos años por la cirugía conservadora del parénquima testicular o la observación, al no haberse asociado su presencia con esterilidad ni malignización.

Realizar un correcto diagnóstico diferencial con tumores testiculares malignos y localizar las frecuentes malformaciones de la vía urinaria alta que a ella se asocian son los dos objetivos fundamentales que deben perseguirse tras su diagnóstico.

\section{REFERENCIAS}

1. Leissring JC, Oppenheimer RO. Cystic dysplasia of the testis: a unique congenital anomaly studied by microdissection. J Urol 1973 Sep;110:362-363.

2. Calleja Escudero J, Pascual Samaniego M, Garrido Redondo M, Matas Gómez V, Fernández Domínguez L, Fernández del Busto E. Lesiones quísticas testiculares en la infancia. Actas Urol Esp 2004;28(8):602-605 
3. Nistal M, Regadera J, Paniagua R. Cystic dysplasia of the testis: light and electron microscopic study of 3 cases. Arch Pathol Lab Med. 1984 Jul;108(7):579-583.

4. Cimador M, Rosone G, Castagnetti M, Libri M, Bertozzi M, Lima $\mathrm{M}$ et al. Cystic dysplasia of the rete testis associated with ipsilateral renal agenesis. Case report. Minerva Pediatr. 2003 Apr; 55(2):175-179.

5. Loo CKC, Yung T. Cystic dysplasia of the testis: a report of three cases and review of the literature. Pediatr Pathol Lab Med. 1995 Nov-Dec;156):885-893.

6. Robson WL, Thomason MA, Minette LJ. Cystic dysplasia of the testis associated with multicystic dysplasia of the kidney. Urology. 1998 Mar;51(3):477-479.

7. Toffolutti T, Gamba PG, Cecchetto G, Talenti E, Tchaprassian Z. Testicular cystic dysplasia: evaluation of three new cases treated without surgery. J Urol. 1999 Dec;162(6):2146-2148.

8. Noh PH, Cooper CS, Synder HM $3^{\text {rd }}$. Conservative management of cystic dysplasia of the testis. J Urol. 1999 Dec;162(6):2145.

9. Kogan SJ. Editorial: Testicular cystic dysplasia. A diagnostic and therapeutic enigma? J Urol. 1999 Dec;162(6):2149.

10. Rubenstein RA, Dogra VS, Seftel AD, Resnick MI. Benign intraescrotal lesions. J Urol. 2004 May;171(5):1765-1772.
11. Emir L, Karabulut A, Agras K, Germiyanoglu C, Ozer E, Erol D. A rare cause of cystic testicular mass in an infant-cystic dysplasia of the testis. Scand J Urol Nephrol. 2001 Apr;35(2):153-155.

12. Casey RG, Stunell H, Buckley O, Flynn R, Torreggiani WC. A unique radiological pentad of mesonephric duct abnormalities in a young man presenting with testicular swelling. Br J Radiol. 2008 Mar;81(963):e93-6.

13. Passarella M, Usta MF, Bivalacqua TJ, Hellstrom WG, Davis R. Testicular-sparing surgery: a reasonable option in selected patients with testicular lesions. BJU International. 2003;91(4): 337-340.

14. Eberli D, Gretener H, Dommann-Scherrer C, Pestalozzi D, Fehr JL. Cystic dysplasia of the testis: a very rare paediatric tumor of the testis. Urol Int. 2002;69(1):1-6.

15. Mac New HG, Terry NE, Fowler CL. Cystic dysplasia of the rete testis. J Pediatr Surg. 2008;43(4):768-770.

Correspondencia autora: Dra. Raquel González López

Servicio de Urología. Hospital Ramón y Cajal

Ctra. Colmenar Viejo km. 9,100 - 28034 Madrid. Tel.: 913368000

E-mail autora: rakelgon@hotmail.com

Información artículo: Revisión - Andrología

Trabajo recibido: julio 2008

Trabajo aceptado: enero 2009 